\title{
ULF Waves from the Ionosphere to the Outer Planets
}

\section{Margaret Galland Kivelson}

Institute of Geophysics and Planetary Physics

UCLA, Los Angeles, CA 90095-1567, USA.

AGU Geophysical Monograph series

K. Takahashi and P.-J. Chi, Editors

UCLA Institute of Geophysics and Planetary Physics Publication Number \#6264 



\title{
ULF Waves from the Ionosphere to the Outer Planets
}

\author{
Margaret Galland Kivelson \\ University of California, Los Angeles, California
}

\begin{abstract}
Space plasma science was launched by studies of ULF waves before in situ measurements became available. Remarkably, interest in the subject has not diminished in the intervening years. This paper addresses selected recent developments related to the subject of ULF waves. Topics touched on include normal modes of the magnetosphere, plasma transport by waves on the magnetopause and the role of ULF waves in relativistic electron acceleration. Waves that may be resonant or driven externally include substorm-associated Pi2 waves and the discrete spectrum of $\mathrm{mHz}$ waves identified in both the ionosphere and the magnetosphere. The outer planets enter through consideration of the structure of mirror mode waves in Jupiter's magnetosheath.
\end{abstract}

\section{INTRODUCTION}

Articles and books on space plasmas almost always start with rhapsodic descriptions of the aurora (Figure 1) and the wonder that it must have inspired in the earliest days of humankind. Then Earth's magnetic field is acknowledged with a reproduction (Figure 2) of Gilbert's magnetized sphere. (I often wonder if I am the only one who is troubled by the prone orientation of Earth's dipole moment.) Some time later comes the first description of the low frequency oscillations of a magnetic needle and its association with auroral activity and later its association with solar activity. It is easy to make the argument that the study of ultralow frequency (ULF) waves is one of the oldest topics in space plasma physics that remains of scientific interest.

The ideas that launched the quantitative study of these waves can be attributed to Alfvén (Figure 3), who gave us the set of equations that describe them, and Dungey (Figure 4) who recognized the structure in space of the waves that we observe at Earth's surface. Alfvén routinely gets credit for his important contribution [Alfvén, 1945 and references therein; Alfvén and Fälthammer, 1963] but there are many who associate Jim Dungey's name only with an oft-copied diagram of the reconnecting magnetosphere. Yet in a few compact papers written in the 1950s and 1960s, Dungey [1954, 1958, 1963a and b] laid out the theoretical concepts that have been elaborated in other work over the decades. $\mathrm{He}$ understood the role of the symmetries of the system and the coupling to the ionosphere and worked out the most important aspects of the problem.

Others who contributed in the early years, too many to be named individually, deserve our thanks. Today we all

6843 Slichter Hall, UCLA, Los Angeles, CA 90095

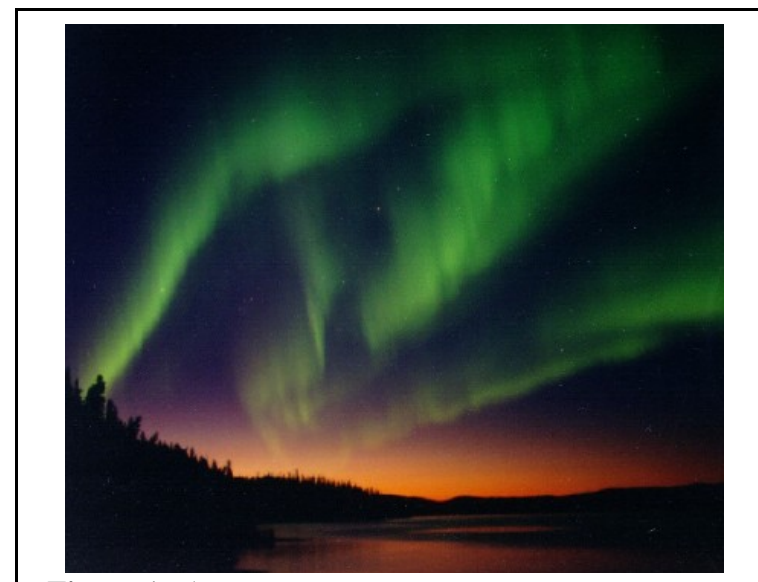

Figure 1. Aurora.

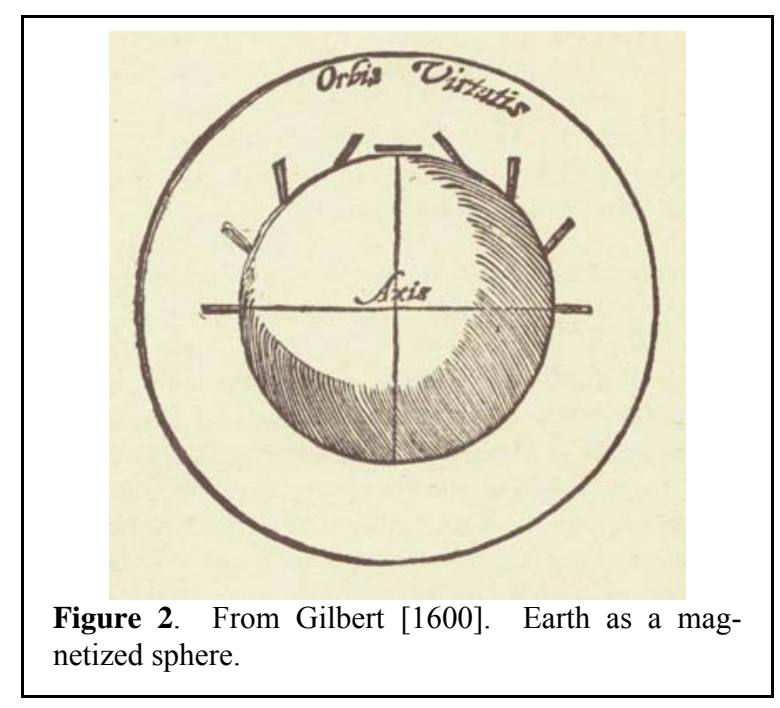


know that ULF waves are excited in diverse ways and specific mechanisms can sometimes be linked to different waveforms and frequency bands that we define as Pc 1-5 or Pi 1-2. The classification introduced in 1964 by Jacobs, Kato, Matsushita, and Troitskaya [1964] helped make sense of what initially was regarded as "lots of wiggles" in the

plotted data. My own interest in ULF waves owes a great deal to the insight and enthusiasm of Valeria Troitskaya (Figure 5) who was far ahead of most of her contemporaries in recognizing the physical significance of the spatial, temporal and frequency distributions of different types of pulsations.

In the years that followed, the characteristics of the magnetospheric pulsations were increasingly well defined. Ground-based observations were soon supplemented by measurements of field and particle modulations in space, which were first interpreted purely theoretically and soon through ever more ambitious simulations. However, it is not the purpose of this paper to review the history of the field but rather to identify some of the issues that are at the forefront of research today. The topics listed in Table 1, extracted from the outline of topics to be covered in this volume, could not be covered in a paper of reasonable length. I shall, therefore, focus on some problems that particularly intrigue me.

Any departure from equilibrium, whether in the field configuration, the plasma pressure distribution, or in unsteady flows, is likely to generate wave disturbances because the magnetosphere is a highly coupled system and changes in a limited spatial region quite generally initiate changes in other parts of the system. Thus it may be difficult to establish the cause of any particular disturbance. Nonetheless, recent years have witnessed extensive efforts to understand how specific types of ULF waves are excited.

One critical driver is the solar wind. The flowing plasma of the magnetosheath can make the magnetopause unstable to the growth of surface waves; these in turn couple to internal resonances by mechanisms that have been understood since the early 1970s [Southwood, 1974; Chen and Hasegawa, 1974]. The surface waves can be amplified by non-linear processes that may enhance the rate of reconnection at certain phases of the wave. An interesting recent analysis of the properties of surface waves (see section 3) recognizes that plasma inhomogeneities along flux tubes just inside the magnetopause can limit the portions of the boundary that are unstable [Hasegawa et al., 2004].

The magnetosphere would probably settle into a quiescent equilibrium if the solar wind were steady, but such is rarely the case. The real solar wind is variable and variation of pressure or field orientation keeps the magnetosphere quivering. Impulsive compression arising from rapid increase of solar wind dynamic pressure requires changes of plasma and field properties throughout the

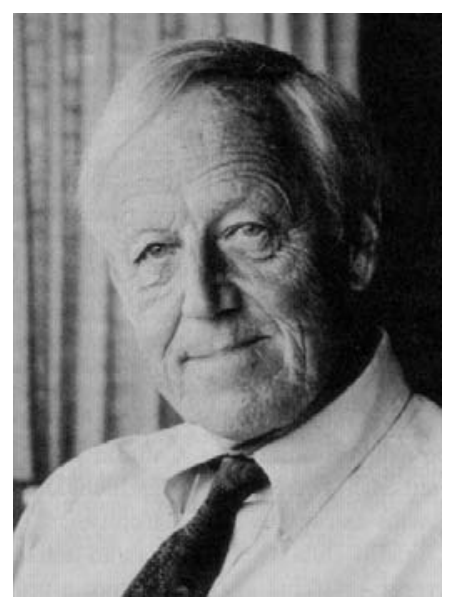

Figure 3. Hannes Alfvén

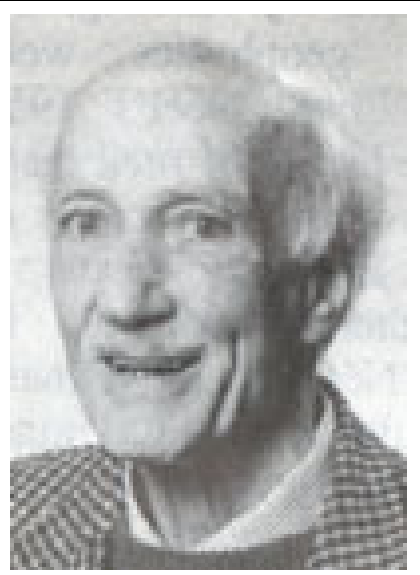

Figure 4. James W. Dungey

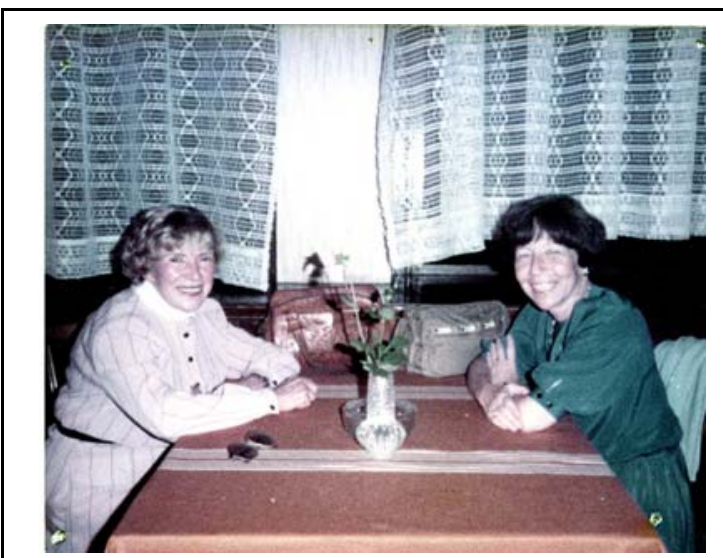

Figure 5. Valeria Troitskaya (left) with the author. Prague (IAGA) 1985 


\begin{tabular}{|l|ll|}
\hline \multicolumn{2}{|l|}{ Table 1. Topics of current interest } \\
\hline $\begin{array}{l}\text { Excitation and } \\
\text { Propagation }\end{array}$ & $\bullet$ & $\begin{array}{l}\text { solar wind momentum } \\
\text { input }\end{array}$ \\
& $\bullet$ & $\begin{array}{l}\text { substorm flows } \\
\text { instabilities and mode } \\
\text { coupling }\end{array}$ \\
\hline $\begin{array}{l}\text { Wave-particle } \\
\text { interactions }\end{array}$ & $\bullet$ & $\begin{array}{l}\text { Drift-bounce resonance } \\
\text { anisotropy-drive }\end{array}$ \\
\hline $\begin{array}{l}\text { Magnetospheric } \\
\text { Diagnostics }\end{array}$ & $\bullet$ & $\begin{array}{l}\text { cross-phase analysis } \\
\text { resonant frequencies }\end{array}$ \\
\hline $\begin{array}{l}\text { New spacecraft or new } \\
\text { techniques }\end{array}$ & $\bullet$ & $\begin{array}{l}\text { waves at outer planets } \\
\text { multi-spacecraft analysis } \\
\text { multi-instrument analysis }\end{array}$ \\
& $\bullet$ & $\begin{array}{l}\text { waves in other } \\
\text { magnetospheres }\end{array}$ \\
\hline
\end{tabular}

magnetosphere. This can excite such normal modes as field line resonances, cavity modes and wave guide modes [Kivelson and Southwood, 1985; Allen et al., 1985; Samson et al., 1992]. Some magnetospheric excitations appear to be directly driven by preferred periodic fluctuations in the wind dynamic pressure [Stephenson and Walker, 2002; Kepko and Spence, 2003].

Internal disturbances also drive waves. Clearly linked to substorm disturbances or other impulsive dynamics are the irregularly shaped waves in the $2-25 \mathrm{mHz}$ band referred to as Pi2. Recent work on these waves suggests that their periodicity reflects the spectrum of global mode excitations of the plasmasphere but there is a competing proposal that the dominant frequencies are imposed by the modulated flows in the magnetotail.

In addition to shear and compressional waves that stand in the magnetosphere or carry energy and momentum from one part of the system to another, the solutions of the gyrotropic form of magnetohydrodynamics include nonpropagating mirror mode waves. Their properties are still being elucidated, and some recent work characterizes the structure of mirror mode waves in Jupiter's magnetosheath.

The role of ULF waves in accelerating electrons to relativistic energies during geomagnetic storms has received a great deal of attention in recent years. It is clear that ULF wave power increases during storms, an anticipated consequence of the non-equilibrium conditions associated with storm-time disturbances. What is less certain is the degree to which ULF wave power plays a controlling role in particle acceleration.

This paper will fill in a few details on the points summarized in this introduction.

\section{CAVITY/WAVEGUIDE MODES}

To those mathematically inclined, it is exceptionally appealing to apply the ideas of normal modes to the magnetosphere. The success of field line resonance theory,

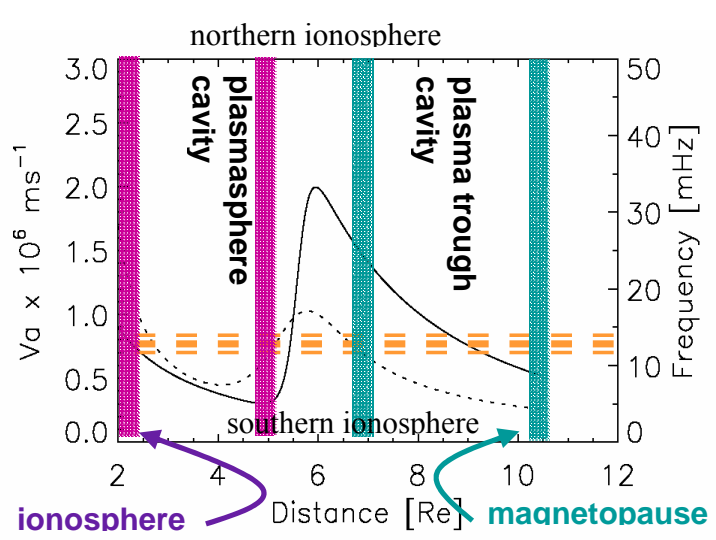

(a)

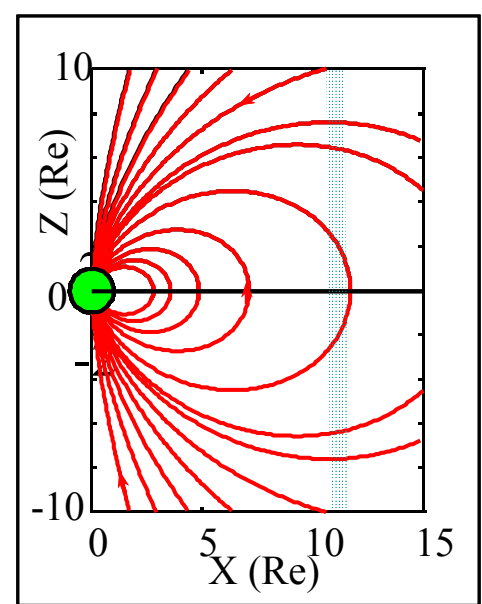

(b)

Figure 6. Theoretical treatments of the cavity mode. (a) The Alfvén velocity in the terrestrial magnetosphere (scale to left) and the corresponding fundamental frequencies of field line resonances (dashed - scale to right) vs. equatorial radial distance from Earth. Pairs of vertical bars define the distances that might bound a cavity mode resonance of frequency indicated by the horizontal dashed line. In the one-dimensional box model the variation of $\mathrm{V}_{\mathrm{A}}$ is achieved by varying the plasma density in a uniform magnetic field bounded by conducting ionospheres. (b) Improved models assume a dipolar field geometry.

which accounts for the spectrum of shear Alfvén waves oscillating on flux tubes, makes it attractive to try to analyze compressional modes in an analogous way. Fast mode resonances can be initiated by a broad band impulse at the magnetopause or in the magnetotail or by driving the cavity at a resonant frequency. Because the fast wave can propagate across (as well as along) the field, excitations can be thought of as standing waves bounded by surfaces at which the Alfvén speed becomes large enough to reflect fast mode waves [Kivelson and Southwood, 1985, 
1986; Allan etc. 1985, 1986]. Beyond the reflection points (or turning points) the cavity mode frequency may match the frequency of a field line resonance (FLR), which can extract power from the cavity wave either through resistive damping owing to the ionospheric boundary conditions or through phase mixing.

Theoretical treatments have been based on either a onedimensional box model (Figure 6a) in which a uniform field or a cylindrical field is bounded by four surfaces. The highlatitude ionospheres at the top and bottom of the box and the low latitude ionosphere at the left end of the box are modeled as reflecting surfaces and the magnetopause at the right is modeled as either reflecting or free. The variation of the Alfvén speed characteristic of the real magnetosphere is obtained by imposing a variation with distance from the earth on the ion density (but not the magnetic field). Solutions include plasma trough resonances with wave power confined outside the plasmasphere and within the magnetopause, plasmaspheric resonances with wave power confined between the plasmapause and the ionosphere, and tunneling solutions (analogous to quantum tunneling) in which both cavities are excited [Zhu et al., 1988]. More sophisticated models [Lee and Lysak, 1991; 1999 for example] use dipolar magnetic geometry and obtain more realistic solutions but the solutions differ little in qualitative structure from the simpler models.

Although analysis has been extensive and most entertaining, the observational evidence for the global mode resonance (monochromatic compressional excitations that cross L-shells) remains limited. Data from ground magnetometer chains at fixed longitude [Samson and Rostoker; 1972] revealed that in Pc4 and 5 events "most micropulsation trains have frequencies that are independent of latitude," as would be expected for excitations driven by a global mode. Reported examples of resonant fast mode waves in the ionosphere and in the magnetosphere are reviewed fully by Waters et al. [2002].

The difficulty in establishing whether resonant fast mode excitations are ever present in the magnetosphere arises because the excitations are likely to be of small amplitude and to decay rapidly. There are several reasons to expect that the amplitude of compressional resonances will be small. Compressional fluctuations are not localized, so wave power readily spreads through a large volume of space. The signals are likely to damp rapidly. In simulations such as those of Lee and Lysak [1991; 1999] and Waters et al. [2002] losses to the ionosphere and to the magnetopause are not well modeled and the amplitudes of the compressional resonances differ only by small factors from those of field line resonances. However, more realistic treatments of fast-mode resonances argue that they decay both by leaking through the magnetopause [Freeman, 2000] or by driving field line resonances from which the wave power is lost through ionospheric dissipation or phase mixing [Crowley et al, 1987; Mann et al, 1995]. Confinement within the magnetosphere requires that the

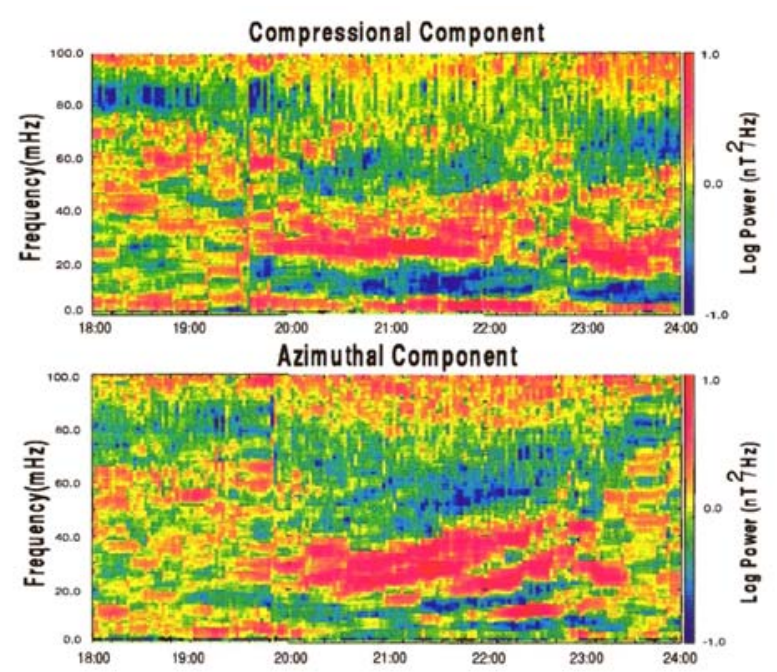

Figure 7. From Kivelson et al. (1997). Power spectrum of fieldaligned and azimuthal components of the magnetic field measured by ISEE 1 on Oct 22, 1977 on an inbound orbit near 10:00 LT that remained within $12^{\circ}$ of the magnetic equator from 20:00 to $24: 00$ UT. The magnetopause crossing occurred at 19:40 UT.

cavity remain stable on the time scale of the pass through the region (hours). However, on the time scale of hours, external conditions are rarely steady. In an example from ISEE 1 measurements, Kivelson et al. [1997] identified compressional power at a roughly constant frequency of 24 $\mathrm{mHz}$ over an interval of several hours during which the spacecraft moved through the outer dayside magnetosphere. The spectrum of the azimuthal component displayed peaks at several harmonics whose frequency increased as L decreased, typical of field line resonances (see Figure 7). The compressional power disappeared for an interval during which the solar wind field orientation rotated from quasi-parallel to the flow to quasi-perpendicular, leading the authors to support a description previously offered by Pilipenko [1990] of the magnetosphere as analogous to a Fabry-Perot interferometer, which absorbs only selected frequencies from an incident source. This interpretation enabled them to conclude from the delay between the rotation of the solar wind magnetic field and the disappearance of the peak in compressional power at 24 $\mathrm{mHz}$ that the time scale for amplitude decay of fast mode resonances is of order 3 wave periods $(Q<3)$. This means that a $24 \mathrm{mHz}$ wave decays in a few minutes, implying that a continuing driver is needed. Field line resonances also must be driven if they are to persist over a time longer than their decay time which is typically $\sim 10$ wave periods.

Waters et al. [2002] identified characteristics of the fast mode resonances in simulations (frequency independent of $L$ over a range of $L$ in the plasmasphere or plasma trough, $90^{\circ}$ phase difference between azimuthal electric and fieldaligned magnetic perturbations, expected nodal structure of 
the compressional magnetic field) and looked for these characteristics in several cases of putative fast mode resonances in the magnetosphere. In the event described by Kivelson et al. [1997], they find no significant maxima in cross phase of the parallel field component evaluated from ISEE 1 and 2 magnetometer data for separations of 400 to $620 \mathrm{~km}$. They acknowledge that it is unlikely that the signature would be detectable for such small separations for the low values of $Q$ that apply in the magnetosphere.

Chi and Russell [1998] report that the phase relation between the electric and magnetic perturbations is a strong function of time and thus incompatible with a standing resonance. However, because the standing resonance decays rapidly, this may not rule out some global mode contribution. As noted above, in the case examined by Kivelson et al. [1997], the putative cavity mode amplitude died away quickly unless the IMF cone angle remained "quasi-parallel" (i.e., $<45^{\circ}$ ) and thus satisfied the condition for transmission of wave power in the Pc3-4 band from the solar wind to the magnetosphere. Intermittent bursts of input power, which need not be coherent, could then account for the temporal variability reported by $\mathrm{Chi}$ and Russell.

One must conclude that although the magnetosphere may wish to ring like a bell, it damps very quickly and must be continually excited. It is unlikely that we will ever detect anything that looks like the cavity/waveguide mode of simulations in spacecraft data. Nonetheless, it is quite likely that cavity/waveguide modes enhance power at resonant frequencies for long enough to drive selected field line resonances (FLRs) and that the detection of a monochromatic frequency band over an interval of hours is possible only if external boundary conditions remain stable. Simultaneous measurements of the magnetic field from multiple radially-aligned spacecraft would be invaluable for testing models of cavity modes and the problem would be a natural objective for a constellation of spacecraft.

\section{WAVES ON THE MAGNETOPAUSE}

Wind over water produces the waves that most of us first encountered on childhood expeditions to the seashore. The foamy white tips arise when the restoring force of surface tension cannot limit wave amplitude (Figure 8). The instability is referred to as the Kelvin-Helmholtz (KH) instability. The $\mathrm{KH}$ instability on velocity shears can also develop in a magnetized plasma. In the plasma, the curvature of the magnetic field, $\mathbf{B}$, provides the restoring surface tension, and if the flow speed is fast enough, the waves grow and the disturbances become non-linear. If the magnitude of $\mathbf{B}$ differs on the two sides of the boundary, the most unstable waves propagate perpendicular to the direction of the larger $\mathbf{B}$.

The simplest mathematical model of the problem supposes that the two fluids, flowing at different speeds, are separated by a tangential discontinuity whose width is infinitesimal. The instability sets in when the velocity

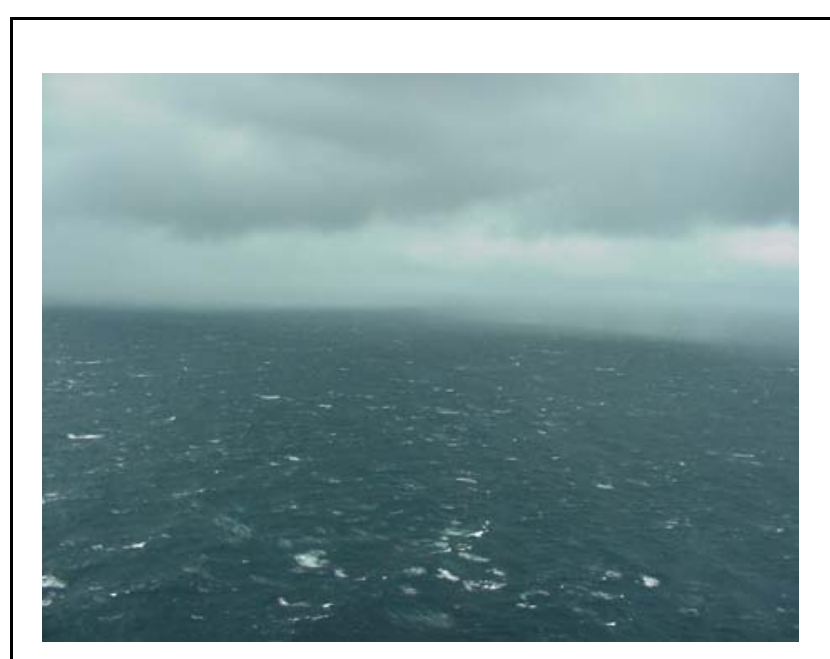

Figure 8. Kelvin-Helmholtz waves on the sea during hurricane Chantal (www.hurricanehunters.com/ chantal.jpg).

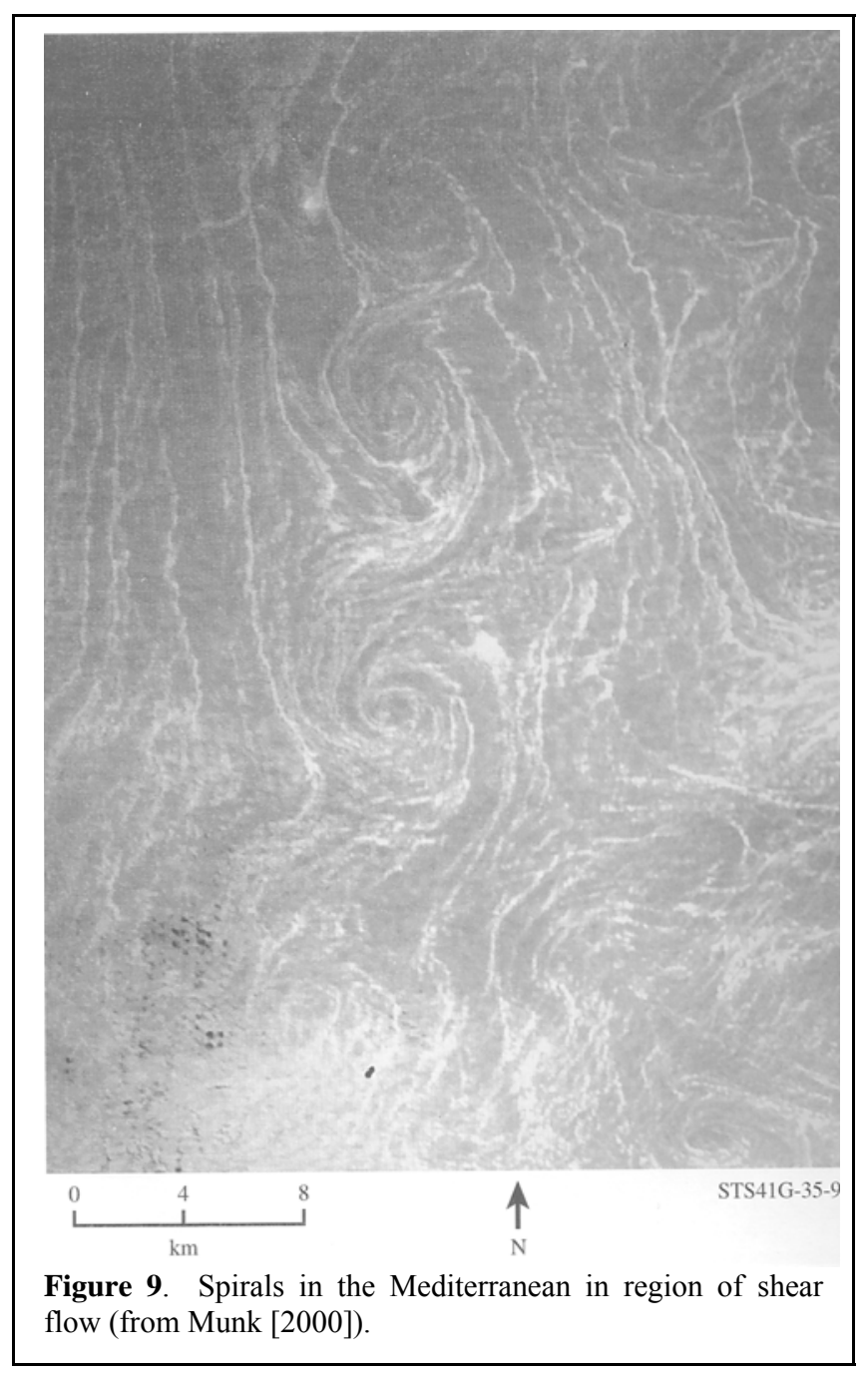




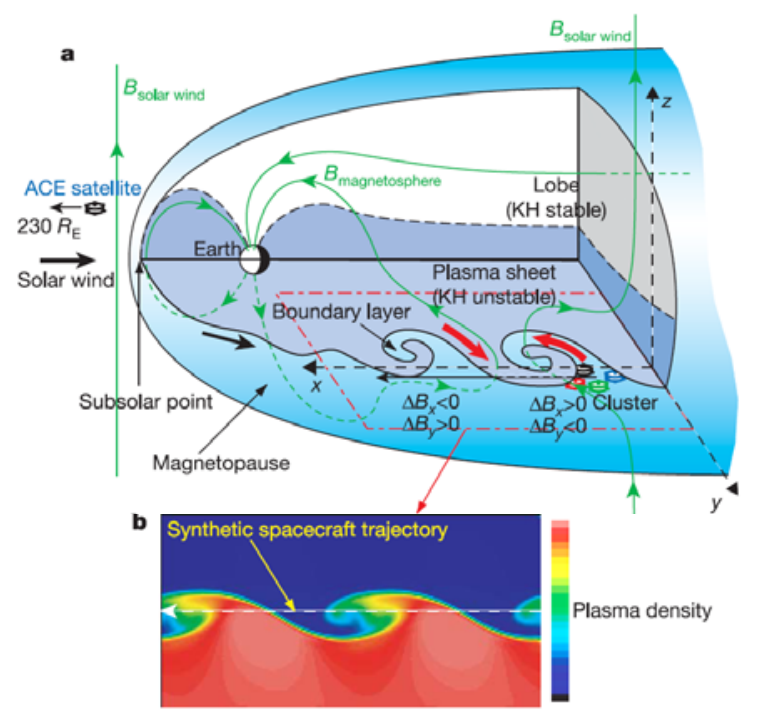

Figure 10. From Hasegawa et al. [2004]. Plasma transported into the magnetosphere at low latitude on the flanks in the presence of $\mathrm{KH}$ boundary waves. The form of the structures is deduced from Cluster data whose trajectory through the surface waves is shown schematically in the lower part of the figure.

difference $(v)$ between the plasma flows on the two sides of the boundary exceeds a critical speed, $v_{c}$ where

$$
v_{c}^{2}=\frac{\rho_{1}+\rho_{2}}{\mu_{o} \rho_{1} \rho_{2}}\left(\left(\mathbf{B}_{1} \cdot \hat{\mathbf{k}}\right)^{2}+\left(\mathbf{B}_{2} \cdot \hat{\mathbf{k}}\right)^{2}\right)
$$

where $\rho$ is mass density, $\mathbf{B}$ is the magnetic field, $\hat{\mathbf{k}}$ is a unit vector in the direction of the wave vector, and subscripts refer to the two sides of the discontinuity.

The model described provides no length scale and consequently the dispersion relation does not establish the wavelength of the growing surface waves. More sophisticated treatments inevitably add at least one length, such as the width of the shear layer separating the two fluids or a velocity gradient scale length. If any length is added to the problem, dimensional analysis tells us that the wave growth will peak for wavelengths of order the assumed length, say within a factor of $\sim 2 \pi$. Thus one must be somewhat skeptical of treatments of the KH problem that identify fastest growing waves. Only if the simulation includes several significantly different relevant length scales (the width of the shear layer, the thickness of the plasma regime on either side of the boundary, the length of the field lines near the boundary) that can be combined to make additional length scales is there a reason to believe that there is a physical significance to the identification of a fastest growing wavelength.

$\mathrm{KH}$ has been invoked in different ways for southward and northward interplanetary magnetic field (IMF) as an intermediary for explaining transport of the magnetosheath plasma into the magnetosphere. For the southward case, $\mathrm{Pu}$

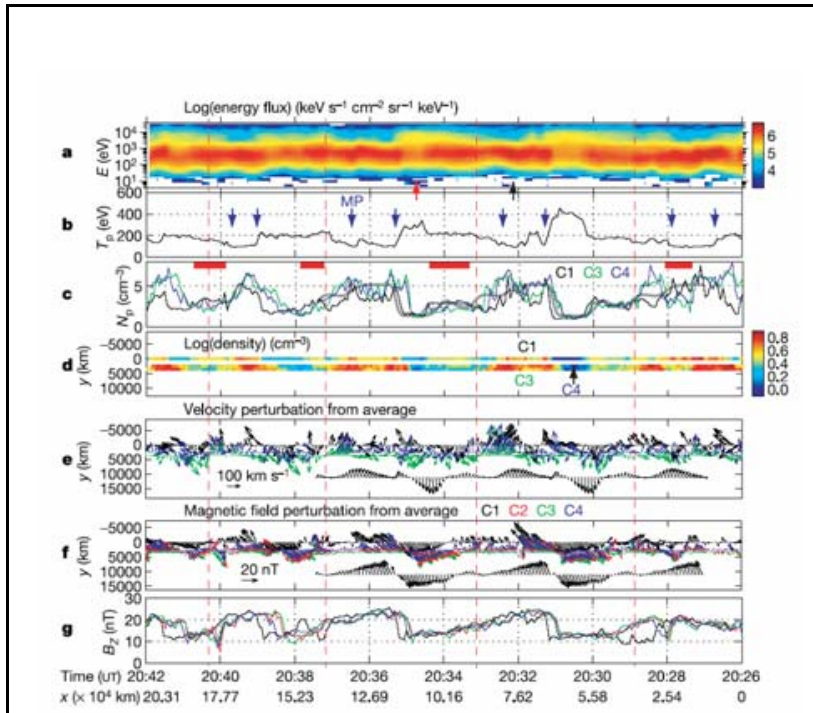

Figure 11. From Hasegawa et al. [2004]. Multi-spacecraft Cluster data from which the schematic boundary motion was inferred.

and his collaborators [Fu et al., 1995; $\mathrm{Pu}$ et al., 1990] among others have modeled cases in which reconnection is facilitated by enhanced resistivity and vorticity in the "white caps" of the unstable waves. This has the appealing feature of accounting for intermittency of reconnection at the dayside magnetopause and the periodic occurrence of FTEs [Lockwood and Wild, 1993] but there is little direct evidence that the process is significant.

Observations of vortical structure that suggest the nonlinear regime of wave growth in the presence of a velocity shear have been imaged in seawater (Figure 9) and also appear to develop on the terrestrial magnetopause. For a case with northward IMF, Hasegawa et al. [2004] used Cluster data to interpret structures that they refer to as "rolled up Kelvin-Helmholtz vortices" at the flank magnetopause (Figure 10). The data from which the structure was inferred are shown in Figure 11 where the flow and field twist as in vortices (panels e and f of Figure 11) and the high density plasma of the magnetosheath penetrates within the magnetopause (panel c of Figure 11). The northward field orientation does not favor reconnection. Instead, Hasegawa et al. propose that magnetosheath plasma is swept into the magnetosphere as tightly wound vortical structures develop on the boundary.

On the magnetopause, the $\mathrm{KH}$ instability is controlled predominantly by magnetospheric conditions. One can argue that the instability condition is most readily satisfied near the equator in the following way. In equation (1), take side 1 as magnetosheath and side 2 as magnetosphere. With $\rho_{1}>\rho_{2} ; \quad\left|\mathbf{B}_{1}\right|<<\left|\mathbf{B}_{2}\right|$, the equation can be approximated as

$$
v_{c}^{2} \approx\left[\left(\mathbf{B}_{1} \cdot \hat{\mathbf{k}}\right)^{2}+\left(\mathbf{B}_{2} \cdot \hat{\mathbf{k}}\right)^{2}\right] / \mu_{o} \rho_{2}
$$




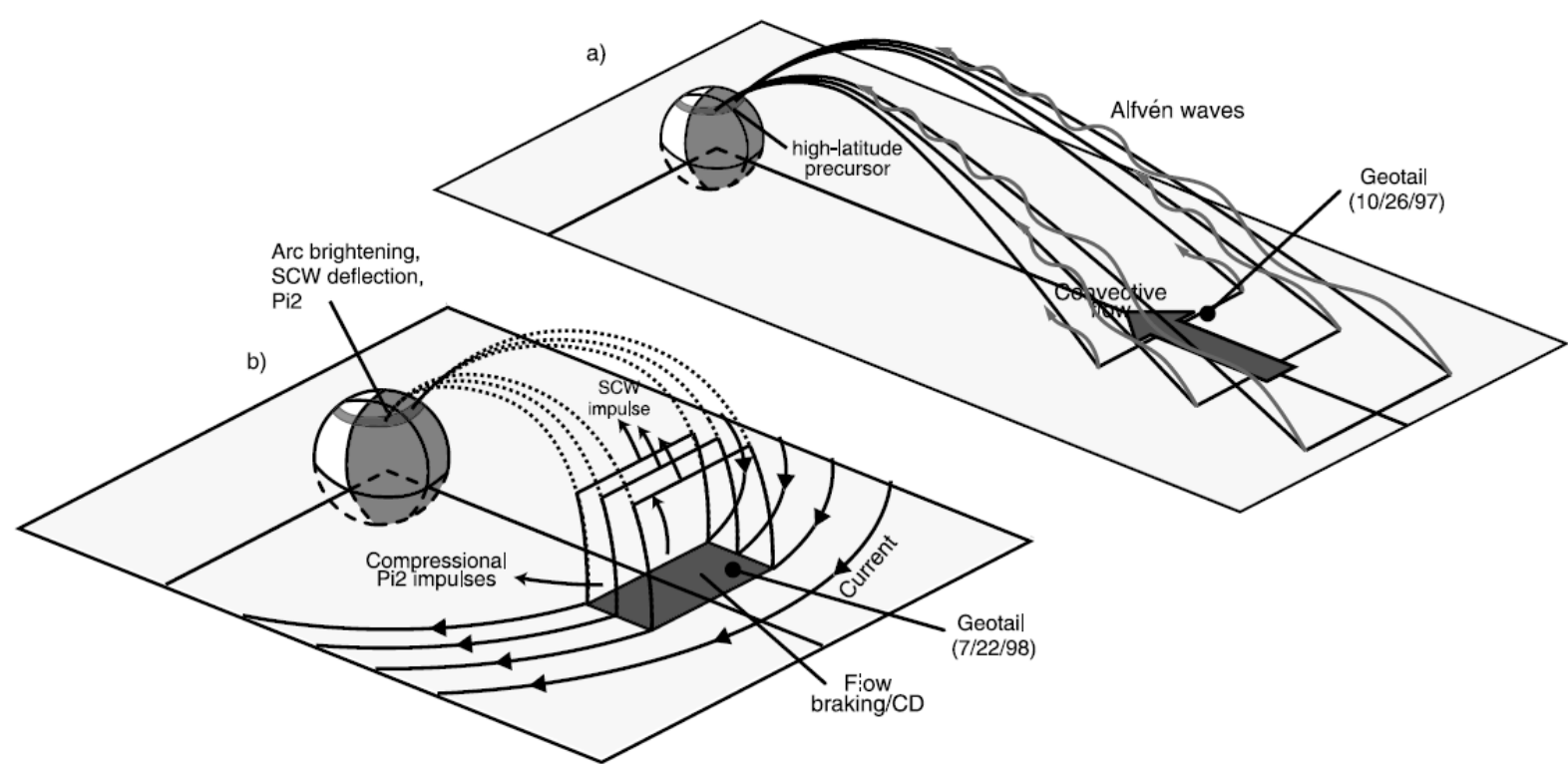

Figure 12. From Kepko et al. [2004]. Schematic showing (a) generation of high latitude Pi2 waves by flow in the plasma sheet at $\sim 10 \mathrm{~s}$ of $\mathrm{R}_{\mathrm{E}}$ down tail which launches Alfvén waves into the ionosphere on flux tubes that shorten with inward convection; (b) deceleration of flow in the quasi-dipolar nightside region and launching of compressional impulses to the inner magnetosphere.

The lowest possible critical speed is found for $\hat{\mathbf{k}}$ orthogonal to $\mathbf{B}_{2}$ in which case one can drop the second term in the final factor of equation (1). The instability condition then becomes

$$
v_{c}{ }^{2} \approx\left(\mathbf{B}_{1} \cdot \hat{\mathbf{k}}\right)^{2} / \mu_{0} \rho_{2}
$$

Thus the onset of instability is controlled by the magnetosheath field and the magnetospheric density.

The magnetosheath field varies little with latitude around the boundary near and beyond the terminator plane but the magnetospheric density along a magnetospheric flux tube near the nightside flanks decreases with distance from the equator. Thus the critical velocity increases in both directions away from the equator along a boundary flux tube. One can easily imagine that the equatorial flux tube will develop surface waves of large amplitude but that the amplitude of the structures shown schematically in Figure 8 decays away from the equator. This would limit the transport process to near-equatorial latitudes. The mechanism could conceivably contribute to the development of the low latitude boundary layer.

\section{DRIVEN WAVES?}

Waves observed in conjunction with substorms (Pi2 waves) and wave power in ground and ionospheric data sets at discrete frequencies well below evident natural frequencies of the magnetosphere with a spectrum that is independent of the state of the magnetosphere [Samson et al., 1991] are sometimes identified as cavity modes. The latter frequencies are sometimes referred to as "magic frequencies". Both phenomena have recently been reexamined in papers that interpret them not as natural magnetospheric resonances but as fluctuations imposed by periodic external forcing. Let us consider the evidence.

\subsection{Pi2 as driven waves}

Pi2 waves by definition fall in the $7-25 \mathrm{mHz}$ frequency band $^{1}$. Although these pulsations have long been investigated, the timing of their appearance relative to substorm onset has not been unambiguously established and just what controls their frequency remains controversial.

Pi2 waves often but not always manifest an irregular waveform at high latitude ground observatories (in the auroral zone). The changing frequencies characteristic of high latitude Pi2s are thought to arise because the waves are generated when earthward flow is initiated in the near-Earth plasma sheet during a substorm. As plasma flows earthward, it perturbs the field in the near-equatorial portion of the high-L flux tube to which it is frozen. This field perturbation launches waves towards the ionosphere. Field line resonance frequencies are thereby excited. As the flux tubes move earthward and shorten, the resonant frequencies increase. This picture (Figure 12a) accounts qualitatively for the waveforms of auroral zone Pi2s and explains why they are systematically linked to substorm-related flows in the magnetotail. The complexity of the waveforms, the sparse distribution of observing stations, the uncertainty in identifying a precise onset time of Pi2 oscillations and the relatively low cadence of global auroral measurements make it hard to establish which starts first: Pi2 waves or

\footnotetext{
${ }^{1}$ The equivalent periods are 40-150s, a range given erroneously in Kivelson and Russell [1995].
} 
related substorm phenomena like auroral brightenings. As Pi2 waves give evidence of the onset of plasma sheet flows, the relative timing of these waves and other phenomena can establish whether substorm expansion is triggered in the tail or closer to the earth, one of the central and most passionately argued issues of magnetospheric physics. The careful timing of the earliest evidence of growth of a Pi2 wave by Kepko et al. [2004] provides strong support for the initiation in the central plasma sheet, but disputes continue.

At low latitudes, the Pi2 waves become quite monochromatic. Explanations of the narrow spectral range of the waves at mid to low latitude are varied. As noted above, some identify the perturbations as normal modes of the plasmasphere and these can be either cavity modes [for example, Sutcliffe and Yumoto, 1991; Allan et al., 1996] or plasmaspheric virtual resonances not fully confined to the plasmasphere [Kim et al., 2005] or linked to the oscillation of a preferred field line resonance. It has also been suggested that they are driven by boundary oscillations on the plasmapause [Sutcliffe, 1975] or some combination of these effects [Cheng et al., 2003]. Polarization analysis shows that the low latitude perturbations are consistent with being driven by compressional hydromagnetic waves launched from a distant source [Itonaga and Yumoto, 1998] as illustrated in Figure 12b. Linked to this interpretation is the suggestion that the frequency of the compressional driver is imposed by a quasi-periodic modulation of flow bursts from the magnetotail [Kepko et al. 2001]. Kepko et al. find close correlation between the velocity of intermittent high speed earthward flows in the near magnetotail (appropriately lagged) and the waveform of Pi2 waves at low latitude ground stations.

If one accepts the proposal of modulated flow from the tail, a new and extremely interesting question arises: what modulates the flow bursts? Various speculations, as yet untested, can be noted. It is possible that flowing plasma heading earthward and slowed on approach to the quasidipolar region generates an inward pressure gradient capable of reflecting some of the earthward-propagating compressional power. This would launch an outwardmoving fast mode wave, which could act on the reconnection site to quench reconnection temporarily. After some time, the transport of flux towards the neutral sheet would produce conditions needed for reconnection to resume. A related possibility is that the onset of reconnection launches compressional power in the northsouth direction and that reflection from the tail magnetopause provides periodic modulation of conditions at the reconnection site. Another possibility is that the kinetic scale instabilities (some form of tearing mode) may have a spatial structure which imposes temporal structure on the flows. These inchoate proposals need fleshing out but suggest that there is yet much to be learned from studies of Pi2 waves.

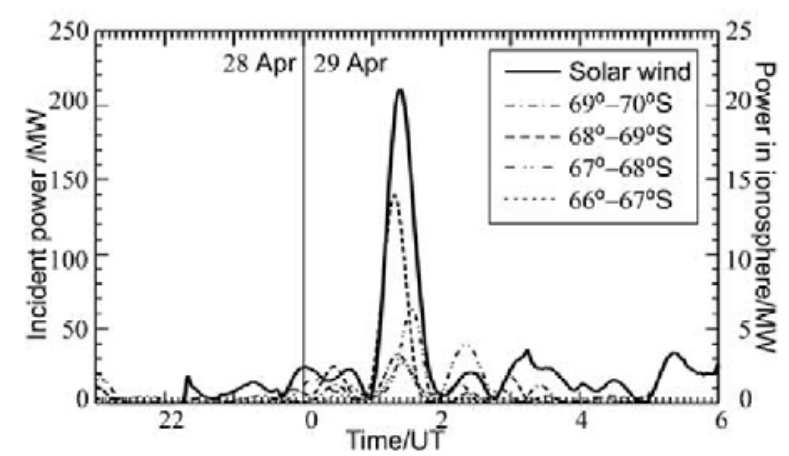

Figure 13. From Stephenson and Walker [2002]: power in the solar wind fluctuations at $3.3 \mathrm{mHz}$ (solid - left axis) and power dissipated in the ionosphere by velocity fluctuations measured by Doppler radar at different latitudes (dotted and dashed - right axis) vs. time.

\subsection{The "magic frequencies" as driven oscillations}

In 1991, Samson et al. [1991] identified a set of frequencies (the magic frequencies) that appear in both magnetometer and radar measurements of ionospheric flows, apparently independent of the state of the magnetosphere. These favored frequencies $(1.3,1.9,2.7$, and $3.3 \mathrm{mHz}$ ) are sometimes referred to as "magic frequencies". Later work has suggested that even lower frequencies $(0.3,0.6$ and $0.9 \mathrm{mHz})$ are present in the discrete spectrum.

Attempts to interpret these frequencies as cavity modes confront some challenges. The lower bound of frequency for nominal conditions in the cavity between the plasmapause and the magnetopause is usually higher than the special frequencies observed, although Samson et al [1991] find that the spectrum at frequencies $\geq 1.3 \mathrm{mHz}$ can be identified as cavity modes between the flank magnetopause and the plasmapause if the magnetopause is at $14.5 \mathrm{R}_{\mathrm{E}}$. To first approximation, the longest wavelength of a cavity mode wave is twice the distance between the inner and outer boundaries $\left(\Delta \mathrm{L} \mathrm{R}_{\mathrm{E}}\right.$ with $\mathrm{R}_{\mathrm{E}}$ the radius of the earth or $\sim 6400 \mathrm{~km}$ ). In the dayside outer magnetosphere, the Alfvén speed near the equator, where number densities are of order $1 \mathrm{~cm}^{-3}$ and the field magnitude is of order 100 $\mathrm{nT}$, the Alfvén speed, is $\sim 1000 \mathrm{~km} / \mathrm{s}$. Then the lowest frequency is

$$
f(\mathrm{~Hz}) \approx 2 V_{A} / \Delta L R_{E} \approx 1 / 3 \Delta L
$$

so, with $\Delta \mathrm{L} \leq 10, f \geq 30 \mathrm{mHz}$. It is hard to imagine dayside conditions that would make this number as small as $\sim 1$ $\mathrm{mHz}$. On the night side where most of the observations have been made, the Alfvén speed decreases and this can lead to a minimum frequency of order $1 \mathrm{mHz}$, but it is more difficult to identify stable boundaries on the night side that have the structure needed to confine waveguide modes. Furthermore, the suggestion that the characteristic frequencies do not change with magnetospheric conditions 

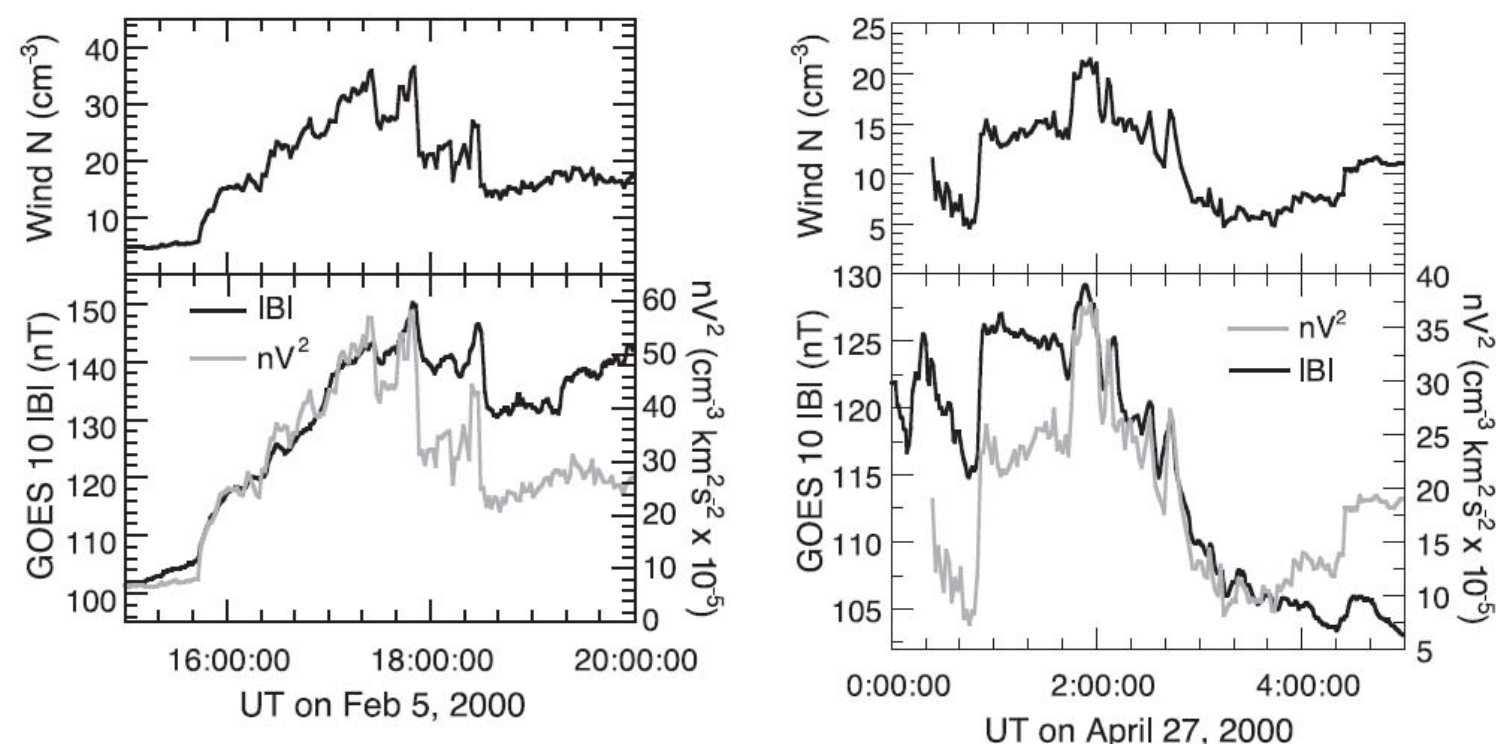

Figure 14. From Kepko and Spence [2003]. Upper panel: Plotted in black, the solar wind density and (b) solar wind dynamic pressure, with scales to the right, vs. UT on Feb. 5, 2000 (left column) and April 27, 2000 (right column). In the lower panels, the field magnitude at GOES 10, a dayside geostationary spacecraft, is also plotted .

is not consistent with cavity/waveguide resonances that depend explicitly on the scale size of the outer magnetosphere.

If the discrete frequency spectrum does not arise through intrinsic resonances of the magnetosphere, the alternative is that the frequency is externally imposed and evidence for such control is mounting. Recently both Stephenson and Walker [2002] and Kepko and Spence [2003] have described cases in which simultaneous measurements in the solar wind and at ground observatories find corresponding spectral peaks in the frequency range between 0.5 and 4 $\mathrm{mHz}$.

Stephenson and Walker [2002] show a case in which there is identifiable power in solar wind fluctuations at and

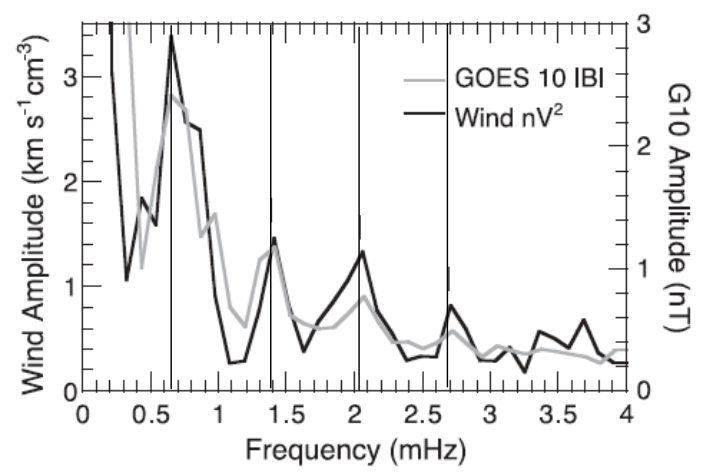

Figure 15. From Kepko and Spence [2003], for the event of Feb. 5, 2000 from 1545 to 1800 UT: Fourier transforms of the solar wind dynamic pressure per ion mass (left axis) and compressional field perturbations (right axis). near the special frequencies of interest. They find (Figure 13) that the power imposed at the magnetopause by solar wind fluctuations in these frequency bands is more than enough to account for the power observed in their magnetospheric counterparts in the night and early morning sectors.

In related work Kepko and Spence [2003] report strong correlations between fluctuations of solar wind density (and dynamic pressure) and compressional fluctuations in the dayside magnetosphere at GOES 10, a geostationary spacecraft. Examples are shown in Figure 14. For the event of Feb. 5, 2000, Figure 15 shows spectra of the solar wind dynamic pressure and the compressional wave amplitude at GOES 10 over an interval of 2.25 hours. Clear peaks appear in both spectra at $\sim 0.6,1.4,2,2.7$ and $3.5 \mathrm{mHz}$, all within $10 \%$ of the magic frequencies. The scale size of the dayside outer magnetosphere is surely less than $10 R_{E}$ and these low frequencies are certainly incompatible with the cavity mode estimate of equation (4).

In agreement with Stephenson and Walker [2002], Kepko and Spence [2003] conclude that the solar wind is the source of the discrete low frequency power observed in the magnetosphere. Now instead of asking why the magnetosphere selects the special frequencies identified by Samson et al. [1991], one needs to ask why these particular frequencies are present in the solar wind. It is not hard to think of links between the solar wind and the solar corona and of links between the corona and the photosphere. The solar photosphere has long been known to show a granular structure with characteristic decay time of 5 minutes and frequencies near $3 \mathrm{mHz}$. Ulrich [1996] identifies them as the source of outgoing Alfvén waves Thompson [2004] 


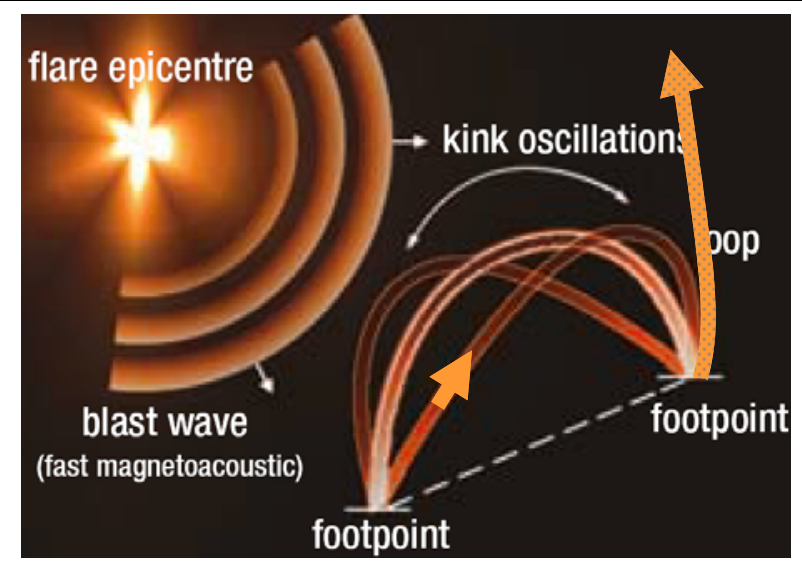

Figure 16. From Morgan et al. [2004]. A representation of the oscillation of an active region on the solar limb imaged by instrumentation on the TRACE spacecraft. Loops oscillate in magnetosonic kink modes with periods of a few minutes as shown in Figure 17.

describes the p-mode spectrum of solar oscillations used for helioseismology as having a peak at $3 \mathrm{mHz}$. Measurements in the corona point to compressive waves with periods of 510 minutes. Nakariakov and Verwichte [2004] report oscillation patterns in the radial expansion of the solar wind whose source is in the corona. Figure 16 shows a schematic of the coronal loop source and Figure 17 reveals that the observed oscillations have periods of 7-8 minutes. Their observations leads us to speculate that the oscillatory pattern in the corona could modulate the reconnection rate in the corona if adjacent coronal loops have oppositely directed magnetic fields. Such modulation of reconnection could readily impose a density variation on the solar wind without greatly affecting the solar wind velocity. Habball and Woo [2004] review additional ways in which coronal variability affects the solar wind.

It is not immediately evident that fluctuating dynamic pressure imposed by the solar wind can compress large portions of the magnetosphere. Thus it is worth confirming that it is plausible for the magnetosphere to breathe in and out at $\mathrm{mHz}$ frequencies. The nominal solar wind speed of $400 \mathrm{~km} / \mathrm{s}$ carries plasma $\sim 3.75 \mathrm{R}_{\mathrm{E}}$ per minute. The periods of the waves discussed above range from 0.6 to $3.5 \mathrm{mHz}$ or 4.8 to 27 minutes. Thus even the shortest period considered gives the solar wind time to flow across $18 \mathrm{R}_{\mathrm{E}}$ in a cycle or $9 \mathrm{R}_{\mathrm{E}}$ in a half cycle. This means that the entire dayside can experience compression and expansion within a cycle of the wave. Global compression at higher frequencies would not be able to impose coherent compressions and expansions, which explains the upper cutoff frequency of the "magic frequency" spectrum.

To summarize, there are many reasons to propose that characteristic frequencies in the $\mathrm{mHz}$ range are present in the outer layers of the sun and that it is the sun itself that imposes $\mathrm{mHz}$ fluctuations on the solar wind. The high correlation between solar wind density variations and the

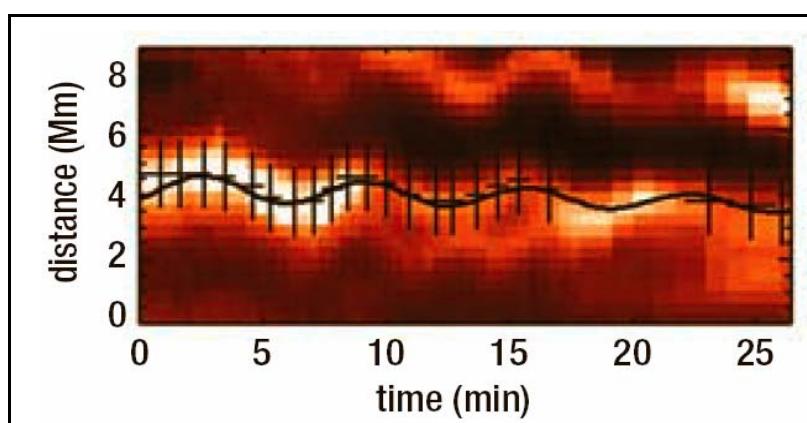

Figure 17. From Morgan et al. [2004]. The kink oscillation with period of $\sim 6$ min of a coronal loop observed by the TRACE spacecraft.

field magnitude at a spacecraft on the day side of the earth makes it clear that the solar wind can impose its dynamic pressure variations on the magnetosphere even at frequencies in the $\mathrm{mHz}$ range. The beauty of this proposal is that, by attributing the underlying frequency content of magnetospheric excitations to the solar wind, it suggests an unanticipated link between the earth's ionosphere and the solar corona that is independent of the sun's radiative energy.

\subsection{Magic frequencies in the plasma sheet?}

If the characteristic discrete spectrum observed in radar and ground magnetometers is imposed by the solar wind, it is reasonable to look for the same discrete spectrum of excitations near $1 \mathrm{mHz}$ in the plasma sheet. To this time there has not been a systematic study of such low frequency excitations of the plasma sheet, but the four Cluster spacecraft dwell in the plasma sheet for many hours on each orbit in the summer season when apogee is in the magnetotail. They are thus well situated to carry out a survey. The Cluster pass on August 15, 2001 gives a hint

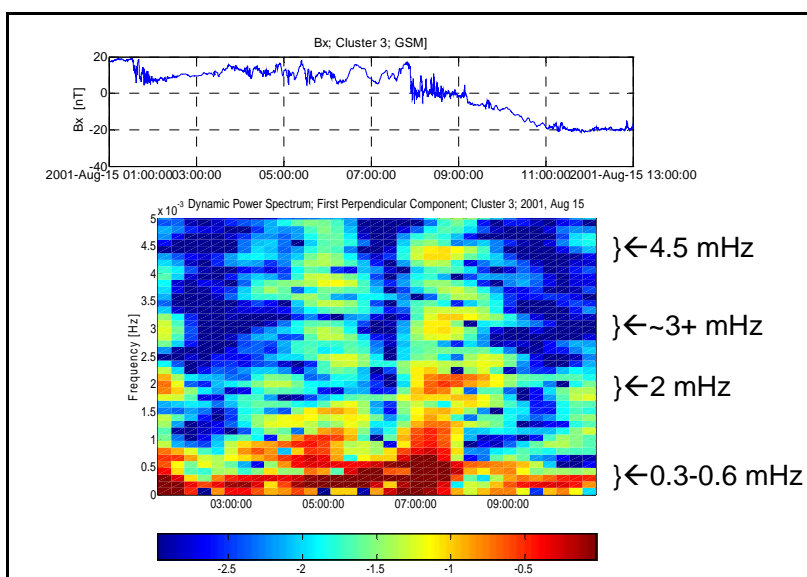

Figure 18. Above: $B_{x}$ in GSM vs. time from 0100 to 1300 UT on August 15, 2001. Below: dynamic spectrum for 0200 to 1000 UT. The Cluster spacecraft remained close to the neutral sheet between 0800 and 1000 during which time discrete peaks appeared in the spectrum near $1 \mathrm{mHz}$. 


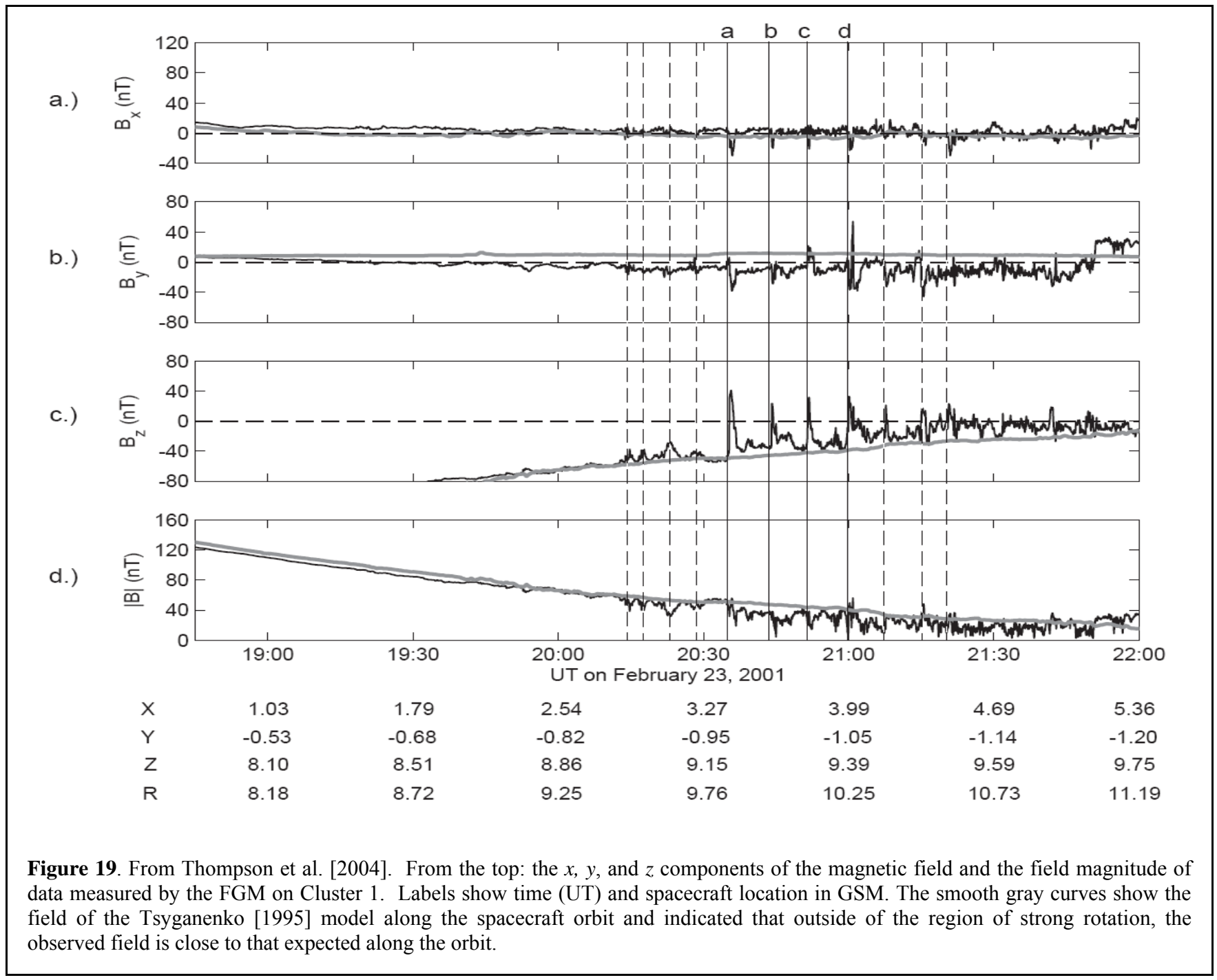

that low frequency waves in the plasma sheet (Figure 18) can have discrete peaks near the key frequencies. The solar wind density measured at ACE (appropriately lagged) shows power in the $\mathrm{mHz}$ range, especially in the range 0.3$0.6 \mathrm{mHz}$ and near $2 \mathrm{mHz}$. A weaker peak appears near 4.5 $\mathrm{mHz}$. This single event suggests that a full study to analyze the link between the solar wind fluctuations and waves in the plasma sheet should be worth pursuing.

\subsection{Other driven oscillations}

If the magnetosphere is subjected to periodic compressional perturbations by the solar wind, it is likely that there are many phenomena modulated at characteristic solar wind frequencies. One may wonder, for example, if the accepted quasi-periodicity of FTE signatures with recurrence periods of $\sim 8 \mathrm{~min}(2 \mathrm{mHz})$ is related to solar wind dynamic pressure modulation.

Possibly related to periodic FTE occurrence is a puzzling set of observations from the Cluster spacecraft poleward of the cusp in the northern lobe of the dayside magnetosphere during an interval of southward oriented interplanetary magnetic field (IMF) [Thompson et al., 2004]. The observations are shown in Figure 19. Periodic large amplitude, short duration field rotations, identified as $a, b$, $c, d$ on the plot, are separated by about 8 minutes. The field, whose magnitude changes little, rotates from $\sim 40 \mathrm{nT}$ southward along the nominal field of the northern dayside lobe to $\sim 40 \mathrm{nT}$ northward and back. A relation to periodic FTEs is possible but there are difficulties in relating the field signatures to dayside reconnection. Because the IMF is southward-oriented, reconnection should take place equatorward of the polar cusp and the reconnected field in regions northward of the cusp should point northward. Attempts to interpret the signature as that of a magnetosheath encounter also run into trouble, even though heated plasma is observed during the interval of northward field. The problem is that one expects to see southward field in the magnetosheath for a southward IMF as 

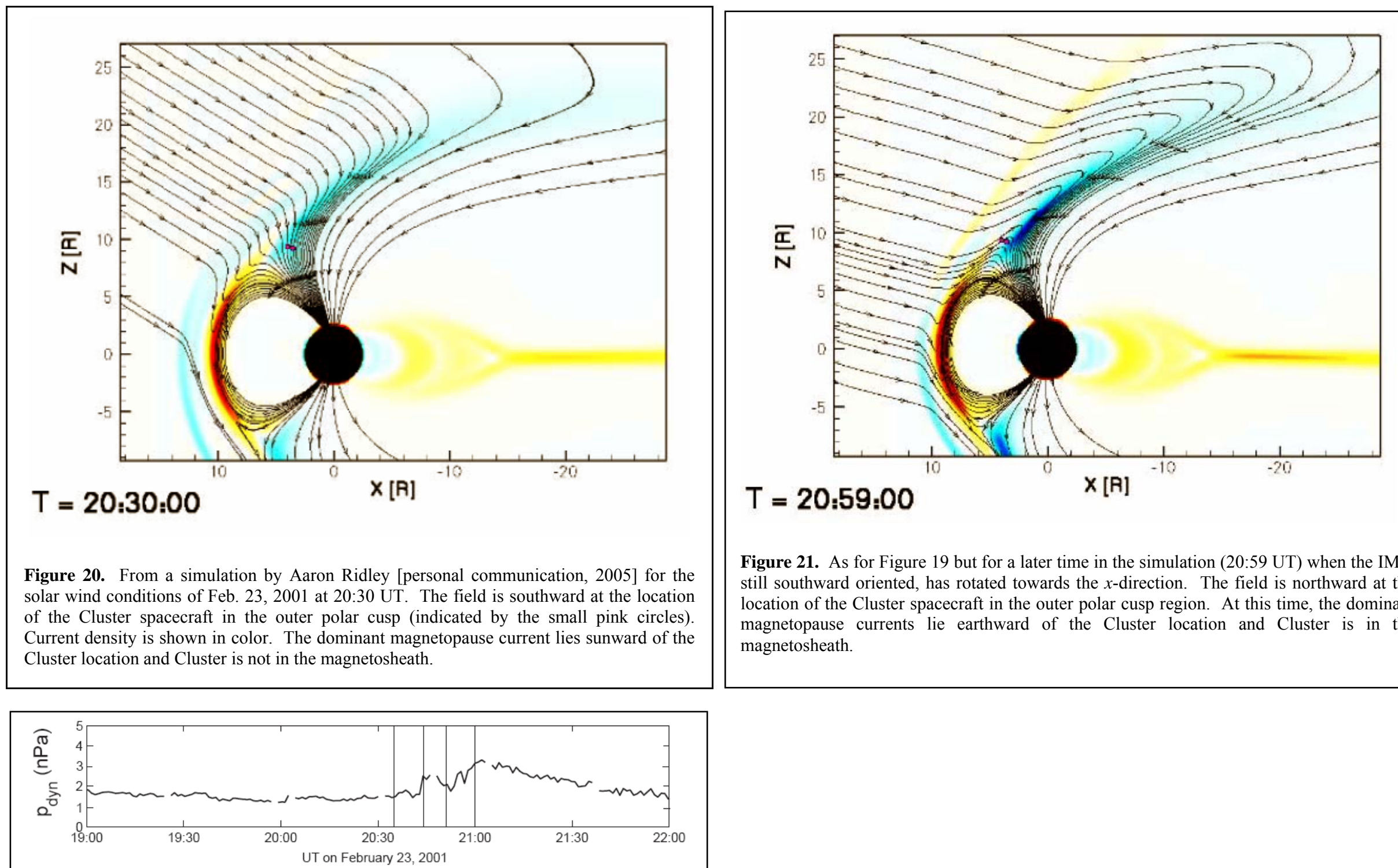

Figure 22. Solar wind dynamic pressure measured by ACE include the interval during which the field rotations were observed at Cluster.

Figure 21. As for Figure 19 but for a later time in the simulation (20:59 UT) when the IMF, still southward oriented, has rotated towards the $x$-direction. The field is northward at the location of the Cluster spacecraft in the outer polar cusp region. At this time, the dominant magnetopause currents lie earthward of the Cluster location and Cluster is in the magnetosheath. 
illustrated in the simulation of Figure 20, yet Cluster observe $B_{z}>0$ in the spikes. (The simulation was kindly carried out for the conditions of the Cluster pass on Feb. 23, 2001 by Aaron Ridley [personal communication 2005].) Ridley's simulation, however, provides an interesting explanation for the observations. He finds that the magnetopause does move in and out by a fraction of an $R_{E}$ even for the small changes in the solar wind dynamic pressure observed (Figure 23). In Figure 21, the peak current (blue) flows outside Cluster's location whereas in Figure 22, the peak current flows inside of Cluster. The boundary motion is seen to account for the intermittent appearance of magnetosheath plasma at the Cluster location.

The most interesting part of the simulation is the effect of small rotations of the IMF on the field structure of the magnetosheath. Figure 20 shows the magnetosphere and magnetosheath at a time when the IMF is significantly tilted southward. Figure 21 shows a later time when the IMF, still with a southward component, has rotated towards the $-x$ direction. Not only has the magnetopause moved inward over the Cluster spacecraft but also the field at its location in the magnetosheath has rotated northward. The explanation is straightforward, though not one that is often considered. As it crosses the bow shock, the solar wind plasma velocity must always rotate away from the local normal. At locations on the bow shock where the angle between the shock normal and the IMF are large, the rotation does not greatly affect the field direction, but when IMF is nearly aligned with the shock normal, the rotation can affect the north-south component of the field. In the simulation, as the field orientation rotates towards the $-x$ direction, the location on the shock where the field aligns with the shock normal moves towards the nose. Everywhere poleward of that location the shocked solar wind rotates northward carrying the field with it. The simulation makes clear that this produces a small but significant region on the day side in which the magnetosheath field is northward even though the solar wind field is southward oriented. The data provide an example of how, in a realistic magnetosphere, combinations of processes may control the response to an imposed periodic perturbation. In such a situation, interpretation may be difficult.

\section{MIRROR MODE WAVES}

Previous sections focused on waves that mediate interactions of magnetospheric plasmas over large portions of the magnetosphere. This section turns to the subject of mirror mode waves, waves that arise from a kinetic instability and play a localized and rather passive role in the system. They grow when significant anisotropy is present in a relatively high $\beta$ background plasma. Here $\beta$ is the ratio of thermal to magnetic pressure. The free energy associated with the anisotropy distorts the magnetic field, providing conditions that create magnetic bottle structures

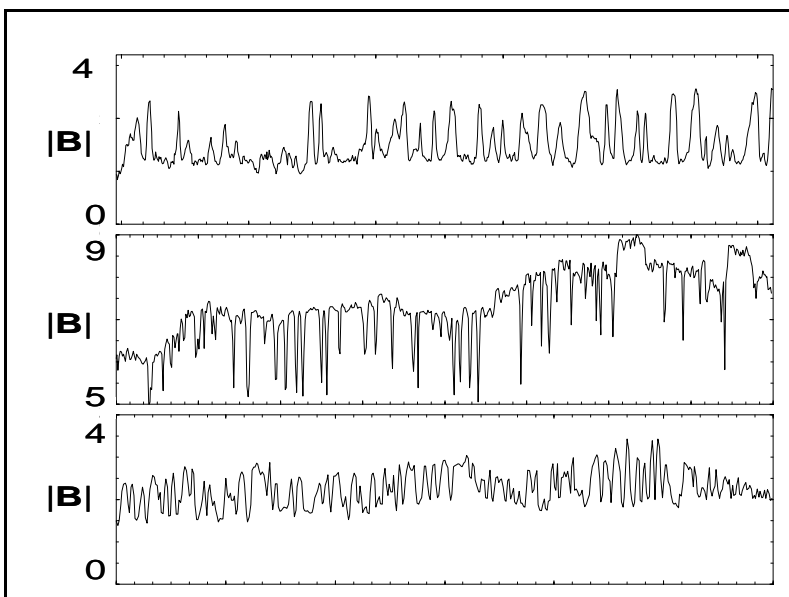

Figure 23. From Joy et al. [in preparation, 2005]. Mirror mode structures observed in Jupiter's magnetosheath by the Galileo magnetometer. The field magnitude in $\mathrm{nT}$ is plotted vs. time for three different days over intervals of four hours.

whose passage over a spacecraft is observed as fluctuations of the field magnitude.

Mirror mode waves do not propagate in the rest frame of a plasma. They appear time-varying to an observer because the plasma is flowing. Samples of mirror mode waves are provided in Figure 23, which shows that the fluctuations of field magnitude are frequently of order the background field magnitude. Possibly it is the large amplitude of the perturbations that continues to attract attention to this wave mode.

The anisotropy condition that supports mirror mode wave growth rate also allows ion cyclotron waves to grow and it has been argued [Gary, 1992] that the latter should dominate the response to plasma anisotropy because the mirror mode wave growth is small compared with that of the ion cyclotron waves. However, the analysis applies in a uniform background field and is invalid when the field magnitude varies along a flux tube. This is because the parallel velocity of ions satisfying the resonance conditions, the ions that supply the energy needed for the waves to grow, depends on the local ion cyclotron frequency. In a uniform field, the latter is constant, so ions resonant over some portion of their field-aligned motion remain in resonance as they move along the field. If, however, the field magnitude varies along the flux tube, ions fall into and out of resonance and ion cyclotron wave growth is strongly inhibited.

Mirror mode wave growth is less sensitive to changes of field magnitude and these waves can grow in a non-uniform background plasma. It seems likely that this is why mirror mode waves are ubiquitous in regions of plasma anisotropy.

When saturated, mirror mode waves are in pressure equilibrium with the sum of magnetic and thermal pressure constant. Slow mode waves are also approximately in pressure balance and, without extensive data analysis, it is not possible to establish unambiguously that the structures 
observed are truly mirror mode waves [see Song et al., 1994]. However, the large amplitude of the field fluctuations combined with the irregularity of the wave form are more likely to be produced by the mirror mode.

It is also possible to interpret the fluctuations of field magnitude as trains of solitons, a proposal that has been promoted by Stasiewicz [2004]. His analysis introduces the assumption $p_{\perp} / N^{\gamma} B^{\kappa}=$ const. This identity reduces to the requirement $p_{\perp} / N B=$ const., i.e. the conservation of the first adiabatic invariant. The latter form follows if both $\gamma$ and $\kappa=1$. However, Stasiewicz's analysis proceeds by setting $\kappa=0$, resulting in $p_{\perp} / N^{\gamma}=$ const., a relation completely independent of the magnetic field. As it seems likely that any large scale non-propagating structure, whether soliton or other, is in pressure balance and the magnetic field contributes to the pressure, it is hard to understand the interpretation provided.

Let us then assume that the waves referred to as mirror mode waves are properly identified. The waveforms plotted in the three panels of Figure 23 differ markedly. The top panel shows intermittent increases of field magnitude, variable in amplitude, riding on top of a slowly varying background field. The middle panel shows intermittent decreases of varying amplitude, dropping below a slowly varying background field. The bottom panel shows fluctuations up and down. It remains unclear what establishes the structure. Joy et al. [personal communication, 2005] are analyzing data from almost all spacecraft that have passed through the Jovian magnetosheath. For the record, this includes Pioneer 10

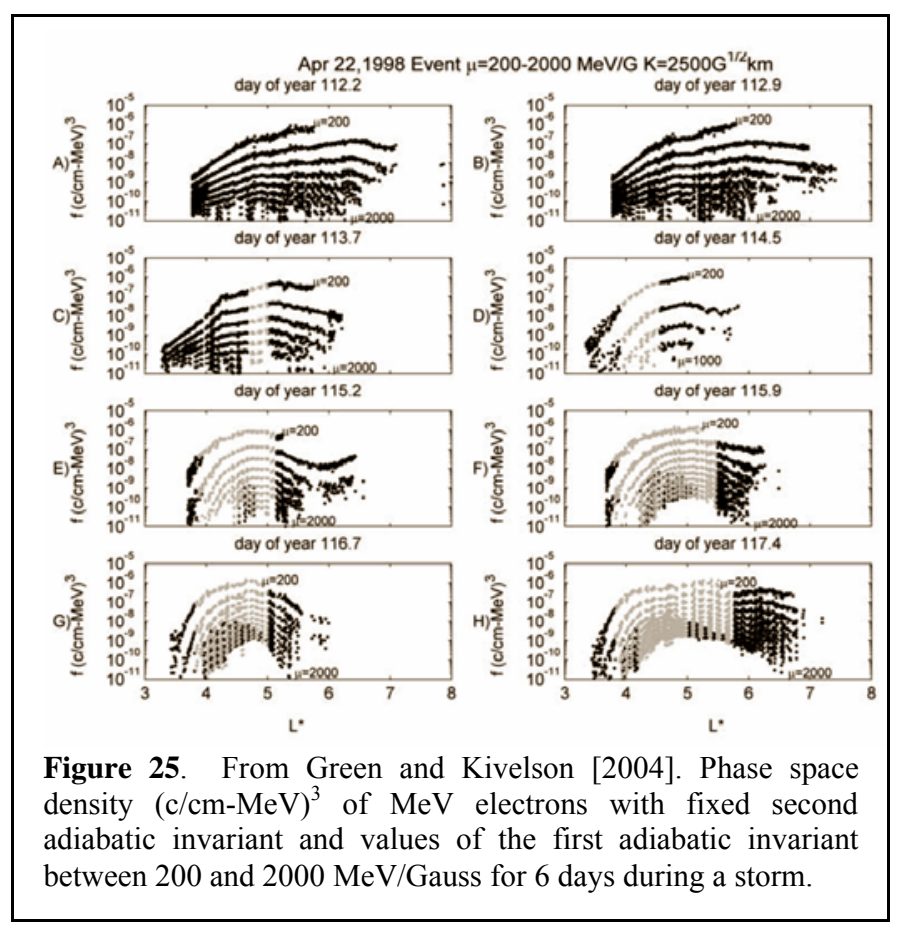

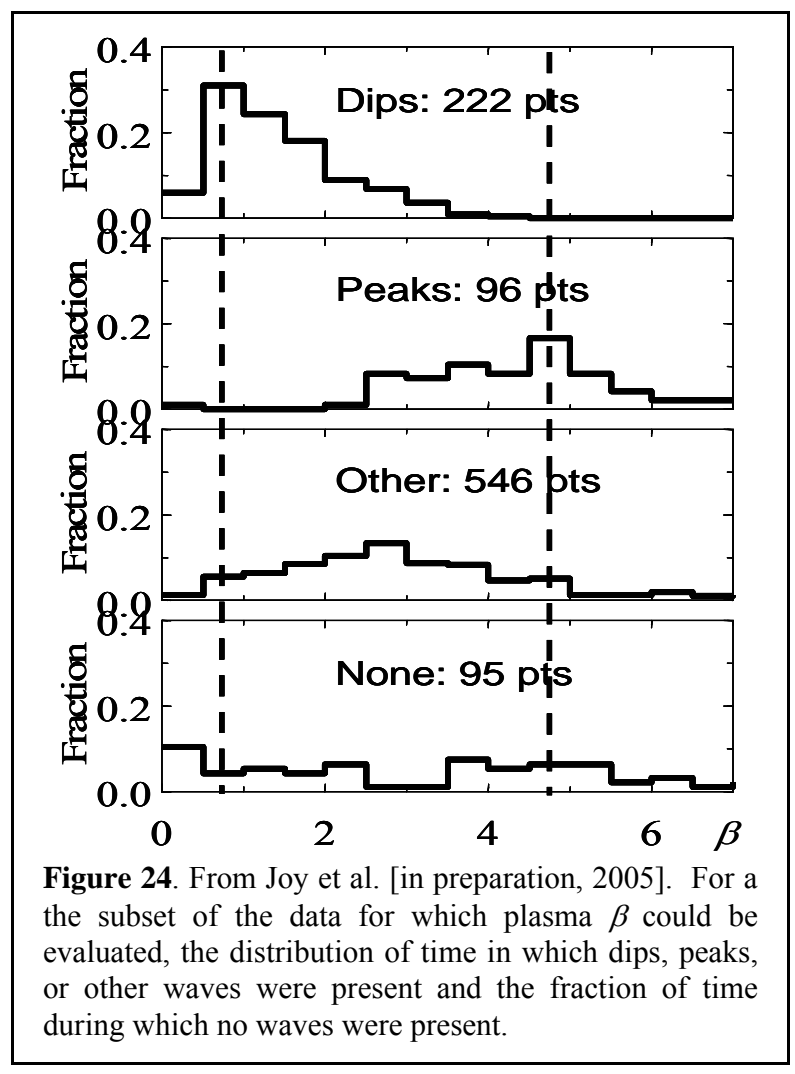

and 11, Voyager 1 and 2, Ulysses, Galileo but not Cassini, which spent little time in the region. Using an automated identification routine, he characterizes the mirror mode waves as peaks, dips, or other. Peaks are intervals in which there are short duration $(<10 \mathrm{~min})$ increases in the magnetic field strength from a clear background level to a random, significantly higher level $(\Delta B>1.25 \mathrm{nT}$ or $\Delta B / B>0.25)$ as in the top panel of Figure 23. Dips appear as field decreases from a slowly varying background field with waveforms similar to those of the middle panel Other mirror mode waves have no well defined background level and fluctuate both up and down, as in the lowest panel.

For a portion of the data set, plasma data from the Galileo PWS investigation, provided by W. Paterson, made it possible to evaluate the plasma $\beta$. Figure 24 shows that dips were especially probable for low $\beta(<1)$, peaks were most probable for high $\beta(\sim 5)$, other waves were common with greatest probability for $\beta$ near 3, and intervals with no waves showed no sensitivity to the value of $\beta$. The peaks are observed most often in regions near local noon, a region in which $\beta$ is higher than in other parts of the magnetosheath

Further work is underway to link the wave forms to the mechanisms generating the anisotropy and possibly to relate changes of structure to the growth of the instability. 


\section{ULF WAVES AND THE ACCELERATION OF RELATIVISTIC ELECTRONS}

As noted in the introduction, considerable attention has been directed to understanding the acceleration of electrons to relativistic energies during magnetic storms. Adiabatic processes are not capable of producing acceleration to $\mathrm{MeV}$ energies, so there seems little doubt that the mechanism must involve wave processes. Studies show that ULF wave power increases during storm times and that the increase precedes the enhancement of relativistic electron flux. It is tempting to assume that the acceleration of the electrons arises through some combination of radial diffusion [Falthammer, 1965] and a resonant acceleration through the action of the fluctuating wave electric field [Elkington et al., 1999]. Mathie and Mann [2000] and O'Brien et al. [2001] have demonstrated correlations between duration of wave power and relativistic electron flux enhancements at geostationary orbit.

Green and Kivelson [2004], abbreviated as GK, converted relativistic electron flux measurements during a storm period into phase space density at constant first and second adiabatic invariant. This allowed them to follow the evolution of the phase space density through the storm period (Figure 25) and to establish the location of its peak. A persistent peak at large $\mathrm{L}$ through the storm interval is required if acceleration by inward radial diffusion is responsible for the appearance of $\mathrm{MeV}$ electrons at $\mathrm{L}<4$ during the storm period. However, for most of the data analyzed, the peak of the phase space density during the storm interval developed between $L$ of 4 and 5 . This result requires acceleration in situ, probably by waves of higher frequency (electromagnetic ion cyclotron waves, etc.) than those discussed in this paper.

Several points should be noted. The GK analysis was based on data acquired well off the equator by the Polar spacecraft. It is possible that it does not represent the processes at work nearer the equator, but this remains to be tested. However, the result is not inconsistent with the earlier evidence [Mathie and Mann, 2000; O'Brien et al., 2001] that ULF wave power of several days duration is associated with increases of $\mathrm{MeV}$ electron flux at geostationary orbit. If the acceleration occurs near $\mathrm{L}=4.5$, producing phase space with peaks inside of geostationary orbit, radial diffusion by ULF waves can spread the energetic electrons towards both higher and lower L (see the lowest right hand panel in Figure 25). Only if the ULF wave power remains enhanced for an extended period will the increase of relativistic electrons be observed at geostationary orbit.

\section{CONCLUSION}

ULF waves continue to fascinate those desirous of understanding dynamics of magnetospheres. They are central to communication among the widely separated parts of the system. Some are normal modes of the system whose structure controls their behavior. Some are sensitive to properties of the internal plasma, its phase space distribution and its $\beta$, others reflect properties of the external plasma, the solar wind. Some accelerate and redistribute the particles trapped within the magnetosphere. This paper has only begun a discussion of the remarkable physics that is revealed through the study of ULF waves, but other contributions to this monograph extend the topics covered here and fill in the all-too-evident gaps.

Acknowledgments. I am grateful to Aaron Ridley of the University of Michigan for running the simulation from which Figures 20 and 21 were taken, to Steven Joy of UCLA for allowing me to use preliminary results from his paper, to William Paterson for providing plasma data used in S. Joy's analysis, to Larry Kepko for his continued interest in ULF wave generation, to Janet Green for teaching me about electron acceleration, to my UCLA colleagues R. L. McPherron, R. J. Walker, K. K. Khurana, J. Weygand, T. King and $\mathrm{H}$ Schwarzl for advice and assistance and to D. J. Southwood for inspiration and challenge. I thank the convenors K. Takahashi and P.-J. Chi for forcing me to think about some interesting material. This work was supported in part by the National Science Foundation under grant ATM 02-05958 and by NASA under grant NNG05GH56G. I am grateful to both agencies for their continued support. UCLA-IGPP Publication \# 6244

\section{REFERENCES}

Alfvén, H., Magneto-hydrodynamic waves and sunspots. I, II, Monthly Notices Roy. Astro. Soc., 105, 3, 1945.

Alfvén, H., and C.-G. Fälthammar, Cosmical Electrodynamics: Fundamental Principles, Oxford University Press, New York, 1963.

Allan, W., S. P. White, and E. M. Poulter, Magnetospheric coupling of hydromagnetic waves--Initial results, Geophys. Res. Lett., 12, 287, 1985.

Allan, W., S. P. White, and E. M. Poulter, Hydromagnetic wave coupling in the magnetosphere-plasmapause effects on impulse-excited resonances, Planet. Space Sci., 34, 371, 1986.

Allan, W., F.W. Menk, B.J. Fraser, Y. Li, and S.P. White, Are low-latitude Pi2 pulsations cavity/waveguide modes?, Geophys. Res. Lett., 23, 765, 1996.

Chen, L. and A. Hasegawa, A theory of long period magnetic pulsations 1 . Steady state excitation of field line resonances, $J$. Geophys. Res. 79, 1024-1032, 1974.

Cheng, C.-C., C.T. Russell, K. Yumoto, Y.F. Gao, and P.J. Chi, Characteristics of consecutive bursts of $\mathrm{Pi} 2$ pulsations observed at the Small Array: A new implication, Geophys. Res. Abs., 5 (1335), 2003.

Chi, P.J., and C.T. Russell, An interpretation of the cross-phase spectrum ofgeomagnetic pulsations by the field line resonance theory, Geophys. Res. Lett., 25, 4445, 1998.

Crowley, G., W.J. Hughes, and T.B. Jones, Observational evidence of cavity modes, J. Geophys. Res., 92, 12,233, 1987.

Dungey, J. W., Electrodynamics of the outer atmosphere, Penna. State Univ. Ionos. Res. Sci. Rept., 69, 1954.

Dungey, J. W., Cosmic Electrodynamics, Cambridge University Press, Cambridge, 1958.

Dungey, J. W., Hydromagnetic waves and the ionosphere, in Proceedings of the International Conference on the Ionosphere, 230, 1963a. 
Dungey, J. W., Structure of the exosphere or adventures in velocity space, in Geophysics, the Earth's Environment, edited by C. DeWitt, Gordon and Breach, New York, 1963b).

Elkington, S.R., M.K. Hudson, and A.A. Chan, Acceleration of relativistic electrons via drift-resonance interaction with toroidal-mode Pc5 ULF oscillations., Geophys. Res. Lett., 26, $3273,1999$.

Falthammer, C.-G., Effects of time-dependent electric fields on geomagnetically trapped radiation, J. Geophys. Res., 70, 2503, 1965.

Freeman, M.P., Effect of magnetopause leakage on the lifetime of magnetospheric cavity modes, J. Geophys. Res., 105, 5463 (doi: 10.1029/1999JA900483), 2000.

Fu, S.Y., Z.Y. Pu, and Z.X. Liu, Vortex-induced magnetic reconnection and sing $\mathrm{X}$ line reconnection at the magnetopause, J. Geophys. Res., 100, 5657, 1995.

Gary, S.P., The mirror and ion cyclotron anisotropy instabilities, $J$. Geophys. Res., 97, 8523, 1992.

Gilbert, W., De Magnete, Dover Publications, Inc., NY, (1958 edition), 1600.

Green, J.C., and M.G. Kivelson, Relativistic electrons in the outer radiation belt: Differentiating between acceleration mechanisms, J. Geophys. Res., 109, A03213, 10.1029/2003JA010153, 2004.

Habbal, S.R., and R. Woo, The solar wind and the Sun-Earth link, Astron. \& Geophys., 45, 4.38, 2004.

Hasegawa, H., M. Fujimoto, T.-D. Phan, H. Rême, A. Balogh, M.W. Dunlop, C. Hashimoto, and R. TanDokoro, Transport of solar wind into Earth's magnetosphere through rolled-up Kelvin-Helmholtz vortices, Nature, 430, 755-58, 2004.

Itonaga, M., and K. Yumoto, ULF waves and the ground magnetic field, J. Geophys. Res., 103, 9285, 1998.

Jacobs, J.A., T. Kato, S. Matsushita, and V.A. Troitskaya, Classification of geomagnetic micropulsations, J. Geophys. Res., 69, 180, 1964.

Kepko, L., and H.E. Spence, Observations of discrete, global magnetospheric oscillations directly driven by solar wind density variations, J. Geophys. Res, 108 (A6), 1257, doi:10.1029/2002JA009676, 2003.

Kepko, L., M.G. Kivelson, and K. Yumoto, Flow bursts, braking, and Pi2 pulsations, J. Geophys. Res., 106, 1903, 2001.

Kepko, L., M. Kivelson, R. McPherron, and H. Spence, Relative timing of substorm onset phenomena, J. Geophys. Res., 109 (A4), A04203, 2004.

Kim, K.-H., D.-H. Lee, K. Takahashi, C.T. Russell, Y.-J. Moon, and K. Yumoto, Pi2 pulsations observed from the Polar satellite outside the plasmapause, Geophys. Res. Lett., 32, L18102, doi:10.1029/2005GL023872., 2005.

Kivelson, M. G. and C.T. Russell, editors, Introduction to Space Physics, Cambridge University Press, New York, 1995.

Kivelson, M. G., and D. J. Southwood, Resonant ULF waves: A new interpretation, Geophys. Res. Lett., 12, 49-52, 1985.

Kivelson, M. G., and D. J. Southwood, Coupling of global magnetospheric MHD eigenmodes to field line resonances, $J$. Geophys. Res., 91(A4), 4345-4351, 1986.

Kivelson, M.G., M. Cao, R.L. McPherron, and R.J. Walker, A possible signature of magnetic cavity mode oscillations in ISEE spacecraft observations, J. Geomag. Geoelectr., 49, 1079, 1997.

Lee, D.-H., and R.L. Lysak, Impulsive excitation of ULF waves in the 3-dimensional dipole model - The initial results, $J$. Geophys. Res., 96, 3479, 1991.
Lee, D.-H., and R.L. Lysak, MHD waves in a three-dimensional dipolar magnetic field: A search for Pi2 pulsations, J. Geophys. Res., 104, 28691, 1999.

Lockwood, M., and M.N. Wild, On the quasi-periodic nature of magnetopause flux transfer events, J. Geophys. Res., 98, 5935, 1993.

Mann, I.R., A.N. Wright, and P.S. Cally, Coupling of magnetospheric cavity modes to field line resonances: A study of resonance widths, J. Geophys. Res., 100, 19, 441, Doi:10.1029/95JA00820, 1995.

Mathie, R.A., and I.R. Mann, A correlation between extended intervals of ULF wave power and storm-time geosynchronous relativistic electron flux enhancements, Geophys. Res. Lett., 27, 3261, 2000.

Munk, W., L. Armi, K. Fischer, and F. Zachariasen, Spirals on the sea, Proc. R. Soc. Lond. A, 456, 1217, 2000.

Nakariakov, V.M., and E. Verwichte, Seismology of the corona of the Sun, Astron. \& Geophys., 45, 4.26, 2004.

O'Brien, T.P., R.L. McPherron., D. Sornette, G.D. Reeves, R. Friedel, and H.J. Singer, Which magnetic storms produce relativistic electrons at geosynchronous orbit?, J. Geophys. Res., 106, 15533, 2001.

Pilipenko, V.A., ULF waves on the ground and in space, J. Atmos. Terr. Phys., 52, 1193, 1990

Pu, Z. Y., P. T. Hou, and Z. X. Liu (1990), Vortex-induced tearing mode instability as a source of flux transfer events, J. Geophys. Res., 95(A11), 18,861-18,869.

Samson, J.C., R.A. Greenwald, J.M. Ruohoniemi, T.J. Hughes, and D.D. Wallis, Magnetometer and radar observations of magnetohydrodynamic cavity modes in the Earth's magnetosphere, Can. J. Phys., 69, 928, 1991.

Samson, J.C., B.G. Harrold, J.M. Ruohoniemi, R.A. Greenwald, and A.D.M. Walker, Field line resonances associated with MHD wave-guides in the magnetosphere, Geophys. Res. Lett., 19, 441, 1992.

Samson, J.C., and G. Rostoker, Latitude-dependent characteristics of high-latitude Pc 4 and Pc 5 micropulsations, J. Geophys. Res., 77 (31), 6133-6144, 1972.

Song, P., C. T. Russell, and S. P. Gary, Identification of low frequency fluctuations in the terrestrial magnetosheath, $J$. Geophys. Res., 99, 6011, 1994.

Southwood, D.J., Some features of field line resonances in the magnetosphere, Planetary Space Science, 22 (3), 483-491, 1974.

Stasiewicz, K., Reinterpretation of mirror modes as trains of slow magnetosonic solitons, Geophys. Res. Lett., 31, L21804, doi:10.1029/2004GL021282., 2004.

Stephenson, J.A.E., and A.D.M. Walker, HF radar observations of Pc5 ULF pulsations driven bythe solar wind, Geophys. Res. Lett., 29, 1297,10.1029/2001GL014291, 2002.

Sutcliffe, P.R., The association of harmonics in Pi 2 power spectra with the plasmapause, Planet. Space Sci., 23, 1975.

Sutcliffe, P.R., and K. Yumoto, On the cavity mode nature of low latitude Pi2 pulsations, J. Geophys. Res., 96, 1543, 1991.

Thompson, M.J., Helioseismology and the Sun's interior, Astron. \& Geophys., 45, 4.21, 2004.

Thompson, S.M., M.G. Kivelson, K.K. Khurana, A. Balogh, H. Reme, A. Fazakerley, L. Kistler, Cluster observations of quasiperiodic impulsive signatures in the dayside northern lobe: High latitude flux transfer events?, J. Geophys. Res., 109, A02213. 10.1029/2003JA010138, 2004. 
Tsyganenko, N.A., Modeling the Earth's magnetospheric magnetic field confined within a realistic magnetopause, J. Geophys. Res., 100, 5599, 1995.

Ulrich, R., Observations of magnetohydrodynamic oscillations in the solar atmosphere with properties of Alfvén Waves, Ap. J., 465, 436, DOI:086/177431, 1996.

Waters, C. L., K. Takahashi, D.-H. Lee, and B. J. Anderson, Detection of ultralow-frequency cavity modes using spacecraft data, J. Geophys. Res., 107(A10), 1284, doi: 10.1029 /2001JA000224, 2002.
Zhu, X., and M. G. Kivelson, Analytic formulation and quantitative solutions of the coupled ULF wave problem $J$. Geophys. Res.,93, 8602, 1988. 